\title{
Research on Four Stations Equipment Safety Evaluation Methods Based on the Combined Enduevt Weight Model
}

\author{
Liu Shenyang, Deng Jian, Wang Lihui, Zhang Junkai \\ Air Force Logistics College, Xuzhou 221000, China
}

Keywords: safety; game theory; set value statistics; evaluation model

\begin{abstract}
The construction of equipment safety is of great significance for improving the safety level of equipment from a deeper level. The evaluation of equipment safety can further promote the effective development of the construction of military equipment safety, and then raise the level of equipment safety management. Aiming at the problem of equipment safety evaluation, a game theory-set value statistical model is proposed. The verification is carried out by examples. The verification results show that the model is scientific and reasonable, simple and practical, and can make an objective and fair evaluation of the level of equipment safety construction.
\end{abstract}

\section{Introduction}

As an important part of the four stations equipment culture, four stations equipment safety culture plays a very important role in ensuring the safety of our stations equipment. As a new stage in the development of the four stations equipment security management, the four stations equipment safety culture can effectively enhance the safety awareness of the four stations equipment of the officers and men of the troops, and make the four stations equipment security become the independent demand of the officers.

For the safety of four stations equipment, the research of Chinese scholars mainly focuses on the connotation and construction measures of four stations equipment safety. There is relatively little research on the quantitative evaluation of the safety construction level of the four stations equipment. Based on the analysis of the connotation and structure of four stations equipment safety, this paper constructs a four stations equipment safety evaluation index system, and puts forward a game theory-set value statistical model to evaluate the level of four stations equipment safety construction.

\section{Equipment Safety Evaluation Index System}

On the basis of science, completeness, independence, and operability, the four stations equipment safety evaluation index system is constructed from the aspects of four stations equipment safety material, four stations equipment safety system, four stations equipment safety behavior, and four stations equipment safety spirit [1]. System of safety evaluation indicators for four stations is shown in Table 1. 
Table 1 System of Safety Evaluation Indicators for Four Stations

\begin{tabular}{|c|c|c|}
\hline \multirow{8}{*}{$\begin{array}{l}\text { Safety evaluation } \\
\text { indicator system }\end{array}$} & \multirow{2}{*}{$\begin{array}{l}\text { Four stations equipped } \\
\text { with safety substance } \\
\mathrm{C}_{1}\end{array}$} & $\begin{array}{l}\text { Four stations equipped with safety equipment } \\
\mathrm{C}_{11}\end{array}$ \\
\hline & & $\begin{array}{llll}\text { Four stations with safe environment } \\
\text { conditions } C_{12}\end{array}$ \\
\hline & \multirow[b]{2}{*}{$\begin{array}{l}\text { Four stations safety } \\
\text { system } \mathrm{C}_{2}\end{array}$} & Four stations safety policy clarity $\mathrm{C}_{21}$ \\
\hline & & $\begin{array}{l}\text { Four stations equipped with security } \\
\text { organization settings } \mathrm{C}_{22}\end{array}$ \\
\hline & \multirow{2}{*}{$\begin{array}{l}\text { Four stations equipment } \\
\text { safety behaviour } C_{3}\end{array}$} & $\begin{array}{l}\text { Four stations equipment safety emergency } \\
\text { disposal simulation exercise status } C_{31}\end{array}$ \\
\hline & & $\begin{array}{l}\text { Four stations equipped with safety education } \\
\text { status } C_{32}\end{array}$ \\
\hline & \multirow{2}{*}{$\begin{array}{l}\text { Four stations equipped } \\
\text { with safety spirit } C_{4}\end{array}$} & $\begin{array}{l}\text { Troops four stations equipped with safety } \\
\text { attitude } \mathrm{C}_{41}\end{array}$ \\
\hline & & $\begin{array}{l}\text { Troops four stations equipped with safety } \\
\text { quality } \mathrm{C}_{42}\end{array}$ \\
\hline
\end{tabular}

\section{Game Theory-Set Value Statistical Models}

\subsection{Game Theory Combination Empowerment Models}

For The weight of the four stations safety evaluation index system directly influences the objective rationality of the four stations safety evaluation results. At present, the methods for determining the weight of indicators can mainly be divided into two types: subjective empowerment method and objective empowerment method [2]. In order to enhance the objective rationality of the evaluation indicators, this paper combines the subjective and objective empowerment methods and uses the game theory assembly model to combine and empower the index weights. The steps for the use of this model [3][4] are as follows:

The $\mathrm{L}$ kinds of methods are used to determine the weight of the evaluation index, and the $\mathrm{L}$ evaluation index weight vector can be obtained: $w(k)=\left[w_{k 1}, w_{k 2}, \cdots, w_{k n}\right]$.

Any linear combination of weight vectors is:

$$
w=\sum_{k=1}^{L} \alpha_{k} w_{k}^{T}
$$

In the equation, wis a weight vector obtained by cross-merging basic weight vectors, and its total is a weight vector set. The weight vector set is

$$
\left\{w \mid w=\sum_{k=1}^{L} \alpha_{k} w_{k}^{T}, \quad \alpha_{k}>0\right\}
$$

The game theory assembly model is essentially a multiplayer optimization problem, then the problem of seeking the most satisfactory weight vector is the optimization of the linear combination coefficient $\alpha_{k}$ in the equation. This in turn minimizes the potential weight vector and the deviation between $w$ and $w_{k}$. The countermeasure model can be derived. It is shown in the equation:

$$
\min \left\|\sum_{j=1}^{L} \alpha_{j} w_{j}^{T}-w_{i}^{T}\right\|(i=1,2, \ldots, L)
$$


From the differential properties of the matrix, it can be obtained that the first derivative condition that can be optimally placed in the equation is

$$
\sum_{j=1}^{L} \alpha_{j} w_{i} w_{j}^{T}=w_{i} w_{i}^{T}(i=1,2, \cdots, L)
$$

$\left(\alpha_{1}, \alpha_{2}, \ldots, \alpha_{L}\right)$ can be obtained from the above formula, followed by normalization, that is

$$
\alpha_{k}^{*}=\frac{\alpha_{k}}{\sum_{k=1}^{L} \alpha_{k}}
$$

The combined weight can be obtained, it is

$$
w^{*}=\sum_{k=1}^{L} \alpha_{k}^{*} w_{k}^{T}
$$

\subsection{Set Value Statistical Models}

The set value statistics method only needs experts to give a rough interval estimate, and can make a quantitative evaluation of each evaluation index. It shows great advantages in the evaluation of the safety culture of the four-station equipment.

Assuming that there are a total of $n$ experts involved in the evaluation of the safety of the fourstation equipment, the interval estimate given by the k-experts for the first evaluation indicator is $\left[x_{i 1}^{(k)}, x_{i 2}^{(k)}\right]$, then the interval estimates given by the $n$ experts for a certain indicator can be formed. The set value statistical sequence is $\left[x_{i 1}^{(1)}, x_{i 2}^{(1)}\right],\left[x_{i 1}^{(2)}, x_{i 2}^{(2)}\right], \ldots,\left[x_{i 1}^{(n)}, x_{i 2}^{(n)}\right]$. The $n$ interval estimates are superimposed, and a distribution covering the valuation axis $X$ can be formed. This distribution can be described by the equation.

$$
\overline{G_{i}}(x)=\frac{1}{n} \sum_{k=1}^{n} G_{\left[x_{i 1}^{(k)}, x_{i 2}^{(k)}\right]}(x)
$$

In the equation,

$$
G_{\left[x_{i 1}^{(k)}, x_{i 2}^{(k)}\right]}(x)=\left\{\begin{array}{lc}
1 & x_{i 1}^{(k)} \leq x \leq x_{i 2}^{(k)} \\
0 & \text { other }
\end{array}\right.
$$

According to the formulas, it can be proved:

$$
\int_{x_{\min }}^{x_{\max }}\left(x-\bar{x}_{i}\right)^{2} \bar{G}_{i}(x) d x=\frac{1}{3 n} \sum_{k=1}^{n}\left[\left(x_{i 2}^{(k)}-\bar{x}_{i}\right)^{3}-\left(x_{i 1}^{(k)}-\bar{x}_{i}\right)^{3}\right] \quad d_{i}=\frac{1}{3} \frac{\sum_{k=1}^{n}\left[\left(x_{i 2}^{(k)}-\bar{x}_{i}\right)^{3}-\left(x_{i 1}^{(k)}-\bar{x}_{i}\right)^{3}\right]}{\sum_{k=1}^{n}\left[x_{i 2}^{(k)}-x_{i 1}^{(k)}\right]}
$$

The smaller the value $d_{i}$ in the equation is, the higher the confidence $u_{i}$ of the $n$ experts in the evaluation of the indicator will be, indicating that the expert's overall grasp of the evaluation of the indicator is greater. On the contrary, the greater the value $d_{i}$ is , the smaller the confidence $u_{i}$ of the $n$ experts in the evaluation of the first indicator will be, indicating that the expert's overall grasp of the evaluation of the indicator is lower. 
Finally, the final evaluation results can be obtained by weighted calculation of the comprehensive evaluation value of individual evaluation indicators. The calculation formula is as follows:

$$
P=\sum_{i=1}^{8} \bar{x}_{i} w_{i}
$$

In this paper, the safety construction level of four-station equipment is divided into four grades according to the size of the weighted comprehensive evaluation value.

\section{Example Analysis}

Taking a force as an example, the safety construction level of four stations is evaluated. First, four experts in related fields are invited to establish a four stations safety evaluation indicator system based on Table 1 on the basis of investigations and analysis of relevant data. The scoring ranges of each indicator $[0,1]$ are given separately.

(1) Determine the weights of the evaluation indicators. First, the weights of each evaluation index are calculated by the AHP and the deviation method respectively, and then the integrated empowerment of the evaluation index is obtained by using the game theory aggregation model.

(2) Calculate the comprehensive evaluation value of evaluation indicator $C_{11}$.

$$
\bar{x}_{C 11}=0.7444
$$

Similarly, the comprehensive evaluation values of indicators $\mathrm{C}_{12}, \mathrm{C}_{21}, \mathrm{C}_{22}, \mathrm{C}_{31}, \mathrm{C}_{32}, \mathrm{C}_{41}$ and $\mathrm{C}_{42}$ can be calculated as $0.74,0.67,0.73,0.77,0.67,0.70$, and 0.75 .

(3) The confidence of the evaluation indicator $\mathrm{C}_{11}$ is calculated as follows:

$$
\begin{gathered}
u_{C 11}=\frac{1}{1+0.0036}=0.9964 \\
d_{C 11}=0.0036
\end{gathered}
$$

Similarly, the confidence levels of the indicators $\mathrm{C}_{12}, \mathrm{C}_{21}, \mathrm{C}_{22}, \mathrm{C}_{31}, \mathrm{C}_{32}, \mathrm{C}_{41}$ and $\mathrm{C}_{42}$ can be obtained as $0.997,0.993,0.993,0.989,0.993,0.985$ and 0.997 respectively. It is easy to find that the confidence of all indicators is greater than or equal to 0.985 , indicating that the experts have a high degree of assurance on these indicators and the consistency of the evaluation is very good.

(4) Calculate the weighted comprehensive evaluation value of all evaluation indicators. The weighted comprehensive evaluation value of the equipment construction level of the four stations of the force is:

$$
P=0.7050
$$

According to Table 2, it can be found that the overall level of construction of the four stations equipment safety is "Fair", so it still needs to be further strengthened. Specific improvement measures can be carried out in conjunction with the comprehensive evaluation of individual indicators. In particular, the level of integrity of the four stations equipment safety regulations is the lowest among all indicators, so it needs to be analyzed and improved in conjunction with the opinions of experts.

\section{Conclusions}

The safety construction of four stations equipment is a systematic project, which involves many elements. The four stations equipment safety evaluation index system is constructed from the safety 
material of four stations, the safety system of four stations, the safety behavior of four stations, and the safety spirit of four stations. Then a game theory-set value statistical model is proposed. The model is analyzed and validated by an example. The results show that the calculation process is simple and the evaluation results are objective and reasonable. The research results of this paper provide a new method for four stations equipment safety evaluation.

\section{References}

[1] Wenshan Li. A Cultural Analysis of the Safety Management of Grassroots Weapons and Equipment[J]. Journal of Equipment Academy, 2014, (01): 124-129.

[2] Qi Cheng. Structural Entropy Method for Evaluating Index Weight Determination[J]. Systems Engineering Theory and Practice, 2010, 30(7): 1225-1228.

[3] Handong Zheng. Study on Multi-index Grey Target Decision Based on Set Statistical Principles[J]. Mathematics in Practice and Theory, 2014, 44(07): 30-38.

[4] Xiaofang Luo. Fuzzy Comprehensive Evaluation Based on Set Statistics and Its Application[J]. Mathematics in Practice and Theory, 2005, 35(9): 42-47. 